\title{
Electron-Electron Interaction in Linear Arrays of Small Tunnel Junctions
}

\author{
K. K. Likharev and K. A. Matsuoka \\ Department of Physics, State University of New York, Stony Brook, NY 11794-3800
}

\begin{abstract}
We have calculated the spatial distribution of the electrostatic potential created by an unbalanced charge $q$ in one of the conducting electrodes of a long, uniform, linear array of small tunnel junctions. The distribution describes, in particular, the shape of a topological single-electron soliton in such an array. An analytical solution obtained for a circular cross section model is compared with results of geometrical modeling of a more realistic structure with square cross section. These solutions are very close to one another, and can be reasonably approximated by a simple phenomenological expression. In contrast to the previously accepted exponential approximation, the new result describes the crossover between the linear change of the potential near the center of the soliton to the unscreened Coulomb potential far from the center, with an unexpected "hump" near the crossover point.
\end{abstract}

Typeset using REVTEX 
Recent theoretical and experimental studies have resulted in considerable progress in understanding correlated single-electron transfer in ultra-small tunnel junctions (for reviews, see Refs. 1-3). These phenomena may be used as a background for a new generation of analog and digital devices. The most common component of single-electronic devices is a one-dimensional array of small tunnel junctions (Fig. 1(a)). Thus it is very important to gain a quantitative understanding of the Coulomb interaction potential, $U(r)$, between single electrons in such an array.

To our knowledge, all previous works on this topic (see, e.g., Ref. ⿴囗十 and references therein) have used a simple model in which the complete matrix $[C]$ of mutual capacitances between conducting "islands" of the array is truncated to tridiagonal form. In this form of the matrix, the only non-zero elements are (a) the diagonal elements $C_{i, i}=C_{o}$, representing the stray capacitances of the islands, and (b) the nearest-neighbor elements $C_{i, i \pm 1}=C$, dominated by tunnel junction capacitances. Electron-electron interaction in the tridiagonal model is described by a simple exponential law 1 目:

$$
U_{t}(r)=U_{t}(0) \exp \left(-m / m_{o}\right)
$$

where $m=r / a$ is the distance between the two electrons, in units of the array period $a$ (i.e., in number of islands). The parameters $U_{t}(0)$ and $m_{o}$ depend on the $C / C_{o}$ ratio, and in the most important limit of $C_{o} \ll C$ :

$$
\begin{aligned}
U_{t}(0) & =\frac{e^{2}}{2 \sqrt{C C_{o}}}, \\
m_{o} & =\sqrt{\frac{C}{C_{o}}} .
\end{aligned}
$$

The tridiagonal model is strictly correct only if the array is placed parallel to, and very near, a conducting ground plane. However, we are not aware of any experiments which actually have used such a configuration. The presence of a ground plane would increase the stray capacitance $C_{o}$, thus cutting off the single-electron soliton radius $m_{o}$ and suppressing the electron-electron interaction at large distances. Thus, it was our goal to describe long linear arrays of realistic geometry without a ground plane. 
If the length scale of the electron-electron interaction within the array is much larger than the array period (i.e., if $m_{o} \gg 1$ ), the electrostatics of the array should not depend strongly on the details of the geometry of its islands. Thus we can model the experimental array geometry, such as the one shown in Fig. 1(a), with an array of cylindrical islands of arbitrary cross section, such as the ones shown in Fig. 1(b) and Fig. 2(a), provided that we keep the same junction capacitance $C=\epsilon S / 4 \pi d$ and array period $a$.

To bring the interaction problem to an analytically calculable form, we can investigate the continuum limit in which the discrete periodic structure is replaced by a continuous dielectric medium (e.g., as shown in Fig. 1(c)). This approximation is valid when the characteristic length of electron-electron interaction within the array is much larger than the array period, i.e. $m_{o} \gg 1$. The effective dielectric constant $\epsilon_{e f}$ of the medium can be found from the requirement that the relation between the average electric field $\langle E\rangle=(1 / a) \int_{0}^{a} E d z$ and average displacement $\langle D\rangle$ is the same in the dielectric model as in the array model:

$$
\epsilon_{e f}=\frac{a}{d} \epsilon=\frac{4 \pi a C}{S}
$$

For the particular case of the dielectric model with circular cross section of radius $R=\sqrt{S / \pi}$ (Fig. 囵(c)), we can find the electron-electron interaction energy $U_{d}(r)$ from the electrostatic potential $\phi(\rho, z)$ induced in the dielectric cylinder by a charge $e$ located at $z=0$ :

$$
U_{d}(r)=e \phi(0, z=r) .
$$

For the relatively large distances we are interested in, $m \sim m_{o} \gg 1$ (i.e. $m a \gg R$ ) the shape of the initial charge is not important, and it is natural to spread it uniformly over a thin disk $(z=0, \rho \leq R)$. The resulting boundary electrostatics problem can be readily solved by the standard Fourier integral expansion:

$$
\phi(0, z)= \begin{cases}\phi(0,0)+\frac{e}{R^{2}}\left[\int_{0}^{\infty} I_{o}(k \rho) A(k) \cos (k z) d k-\frac{2}{\epsilon_{e f}}|z|\right], & \rho \leq R, \\ \int_{0}^{\infty} K_{o}(k \rho) B(k) \cos (k z) d k, & \rho \geq R,\end{cases}
$$


where the last term in the upper line takes care of the boundary condition (Gauss's law) on the charged disk at $z=0$. On the other hand, the boundary conditions at $\rho=R$ $\left(\partial \phi /\left.\partial z\right|_{+}=\partial \phi /\left.\partial z\right|_{-}, \partial \phi /\left.\partial \rho\right|_{+}=\epsilon_{e f} \partial \phi /\left.\partial \rho\right|_{-}\right)$give

$$
A(k)=\frac{4}{\pi \epsilon_{e f} k^{2}}\left[I_{o}(k R)+\epsilon_{e f} \frac{I_{1}(k R) K_{o}(k R)}{K_{1}(k R)}\right]^{-1} .
$$

[For numerical calculation of the integrals in Eqs. (5), (6), it is convenient to use the expansion $|z| \equiv(2 / \pi) \int_{0}^{\infty} \cos (k z) k^{-2} d k$, in order to cancel the divergence of $A(k)$ at $k \rightarrow 0$.]

Numerical integration yields the functions $U_{d}(r)$ as shown in Fig. 3. At large distances, these functions approach the free space Coulomb interaction:

$$
U_{d}(r) \rightarrow \frac{e^{2}}{r}, \quad r \rightarrow \infty
$$

while at small distances, these functions are linear:

$$
\begin{aligned}
U_{d}(r) & \rightarrow U_{d}(0)-e E(0) r, \quad r \rightarrow 0, \\
E(0) & =\frac{2 e}{\epsilon_{e f} R^{2}} .
\end{aligned}
$$

The crossover between these two limits takes place at $r \simeq r_{o} \equiv R \sqrt{\epsilon_{e f}}$. Quite unexpectedly, at $r \simeq r_{o}$ the potential $U_{d}(r)$ is higher than the asymptotic value (7), approaching it at $r \rightarrow \infty$ from above.

If $r_{o} \gg a$, we can apply this result to the discrete array of a circular cross section. In terms of the number of islands, the crossover point is

$$
m_{o}=\frac{r_{o}}{a}=\frac{R}{a} \sqrt{\epsilon_{e f}}=\sqrt{\frac{4 C}{a}} .
$$

If we define the stray capacitance per unit period of the array as

$$
C_{o} \equiv \frac{a}{4}
$$

we can once again formally express $m_{o}$ as in the tridiagonal model, $m_{o}=\sqrt{\frac{C}{C_{o}}}$.

The dielectric-model potential function $U_{d}$ can be approximated reasonably well by a simple phenomenological expression: 


$$
U_{a}(m)=\frac{e^{2}}{a}\left\{\frac{\alpha}{m_{o}} \exp \left(\frac{-\kappa m}{m_{o}}\right)+\frac{1}{m}\left[1-\exp \left(\frac{-\kappa m}{m_{o}}\right)\right]\right\} .
$$

with two dimensionless fitting parameters, $\alpha$ and $\kappa$. The boundary condition (8), together with the definition for $m_{o}$ given in Eq. (9), yields the condition $\alpha=2 / \kappa-\kappa / 2$, leaving only one free parameter, $\kappa$. Figure 1 shows the dependence of $\kappa$ on $m_{o}$, when $\kappa$ is adjusted to provide the best fitting (by sight) of $U_{a}(m)$ to $U_{d}(m)$ (see Fig. 3). One can see that $\kappa$ is close to unity, and depends on $m_{o}$ only logarithmically, so that

$$
U_{a}(0)=\frac{e^{2}(\alpha+\kappa)}{m_{o} a} \simeq \frac{2 e^{2}}{a} \sqrt{\frac{C_{o}}{C}}=\frac{e^{2}}{2 \sqrt{C C_{o}}} .
$$

Thus, $U_{a}(0)$ may be approximated by $U_{t}(0)\left(\right.$ Eq. (2)) if $m_{o}$ is within the practical interval $\sim 3-10$ (see below).

In order to check the validity of our results for structures with a different cross section, we have calculated the interaction energy $U_{s}(m)$ for a chain of square cross section islands (Fig. 2(a)) for several values of the $a / d$ ratio using the geometric capacitance modeling program FASTCAP目. This program takes, as input, a collection of "panels," finite elements representing the surfaces of a group of conductors. By calculating the amount of charge induced on each panel when one conductor is held at fixed potential, the rest at zero, FASTCAP calculates, one by one, each row of the capacitance matrix for the group of conductors.

For FASTCAP to calculate the capacitances accurately, the discretization of the surface must be fine enough to represent the charge distribution. The main criterion, when panels (and computer memory) are limited, is to let the panelling reflect the increased charge density near the corner of a conductor. Thus, when modeling conductors with square cross section, each face was divided into 9 panels (Fig. 2(b)), with smaller panels along the edges where charge density is higher. To test the accuracy of this simple panelling, we modeled a chain of 10 islands in two ways: one with 9 panels per face, the other with 100 per face. Differences in electron-electron interaction energies between the two models were less than $1 \%$, leading us to believe that the 9-panel model is sufficiently accurate for our purposes in this work. 
Island chains modeled with FASTCAP were limited in length to around 120 islands, due to computer memory limitations. To avoid edge effects, the energy of interaction between two electrons was calculated with both electrons separated from the edges of the array by at least $\sim 2 m_{o}$ islands. Energies calculated this way did not depend significantly on the total number of islands in the chain. The results of the FASTCAP calculations are shown as points in Fig. 3. One can see that they are very close to those obtained by the dielectric model, and hence to the analytical approximation of Eq. (11), despite a substantial difference in the geometry of the two cross sections.

To summarize, we have shown that electron-electron interactions in long one-dimensional arrays of small tunnel junctions are much better described by Eq.(11) (with $\kappa \simeq 1, \alpha \simeq 2$, and $\left.U_{a}(0) \simeq e^{2} / 2 \sqrt{C C_{o}}\right)$ than by the traditional expression (11). The effective radius $m_{o}=$ $\sqrt{C / C_{o}}$ of the interaction is determined by the ratio of the tunnel junction capacitance $C$ to the effective stray capacitance $C_{o}(10)$. For an array formed on a substrate with dielectric constant $\epsilon_{s}$, Eq. (10) should be modified to

$$
C_{o} \simeq \frac{\left(\epsilon_{s}+1\right) a}{8}
$$

For a typical present-day single-electronic array with $a \simeq 0.1 \mu m$, formed on a $\mathrm{SiO}_{2}$ substrate (see, e.g., Ref. (7), the formula above gives $C_{o} / a \simeq 10^{-16} \mathrm{~F} / \mu \mathrm{m}$ and $C_{o} \simeq 10^{-17} \mathrm{~F}$. Thus, for the typical junction capacitance $C \simeq 10^{-16} \mathrm{~F}$, the soliton radius $m_{o}$ is close to 3 . This estimate shows that for single-electronic devices where a considerable soliton radius is important (for example, for suppression of the macroscopic quantum tunnelling2 23 ), vertical structures with stacked junctions may be more advantageous. Vertical stacking can provide a very small array period $a$, and hence the small stray capacitance $C_{o}$ needed for $m_{o} \gg 1$.

The authors are grateful to J. White and K. Nabors for providing the program FASTCAP, and to D. Averin, A. Korotkov and J. Lukens for numerous discussions. This work was supported by AFOSR Grant No. 91-0445. 


\section{REFERENCES}

${ }^{1}$ K. K. Likharev, IBM J. Res. Devel. 32, 144 (1988).

${ }^{2}$ D. V. Averin and K. K. Likharev, in Mesoscopic Phenomena in Solids, edited by B. Altshuler, P. A. Lee, and R. A. Webb (Elsevier, Amsterdam, 1991), p. 173.

${ }^{3}$ Single Charge Tunneling, edited by H. Grabert and M. H. Devoret (Plenum, New York, 1992).

${ }^{4}$ P. Delsing, in Ref. 匂, p. 249.

${ }^{5}$ K. K. Likharev, N. S. Bakhvalov, G.. S. Kazacha, and S. I. Serdyukova, IEEE Trans. Magn. 25, $1436(1989)$.

${ }^{6}$ K. Nabors, S. Kim, and J. White, IEEE Trans. on Microwave Theory and Techniques, 40, 1496 (1992).

${ }^{7}$ P. D. Dresselhaus, L. Ji, S. Han, J. E. Lukens, and K. K. Likharev, Phys. Rev. Lett. 72, 3226 (1994). 


\section{FIGURES}

FIG. 1. One-dimensional array of tunnel junctions: (a) a typical experimental geometry, with tunnel junctions of area $S$ and permittivity $\epsilon$; (b) cylindric island model; and (c) dielectric approximation to cylindric islands.

FIG. 2. (a) Square cross section island array model. (b) Boundary elements ("panels") for square cross section island model used for in FASTCAP capacitance calculations.

FIG. 3. Functions $U(m)$ describing the shape of the single electron soliton. Solid lines: $U_{d}(m)$, dielectric approximation to circular cross section islands, with $\epsilon_{e f}=30,100,300$ (bottom to top). To compare numerical and analytical results, we have set $\pi R^{2}=a^{2}$, so that $m_{o}=\sqrt{\epsilon_{e f} / \pi}$. Squares: $U_{s}(m)$, square cross section array for the case $a=b$ (see Fig. 2). Dashed lines: $U_{a}(m)$, as given by Eq. (11). Dotted line: free space potential, $U(m)=e^{2} / m a$. Note that a vertical offset is used in plotting the curves.

FIG. 4. The best-fit parameter $\kappa$, the sum $\kappa+\alpha$, and $U(0)$, as functions of $m_{o}$. Squares: $U_{s}(0)$. Open circles: $U_{d}(0)$. Diamonds: $U_{a}(0)$. Error bars reflect the range over which a "good" fit, by sight, is achieved. 

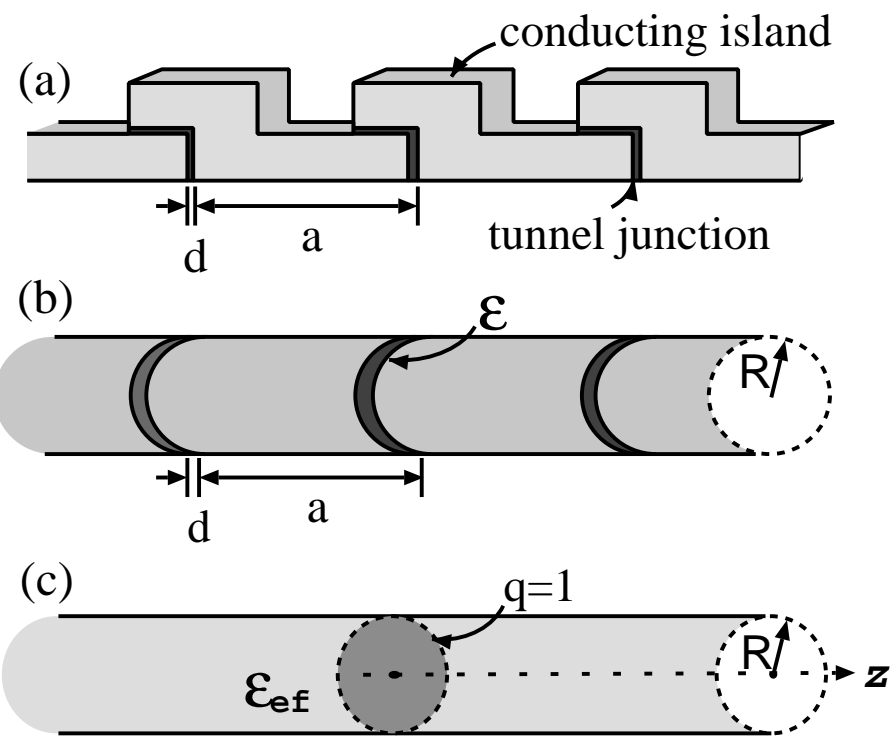

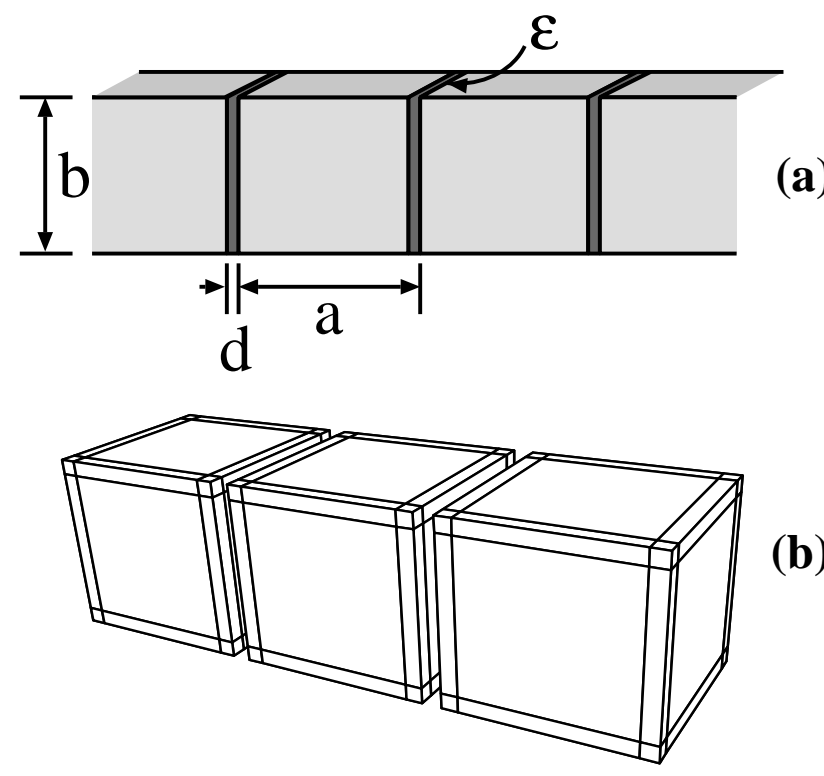

(b) 


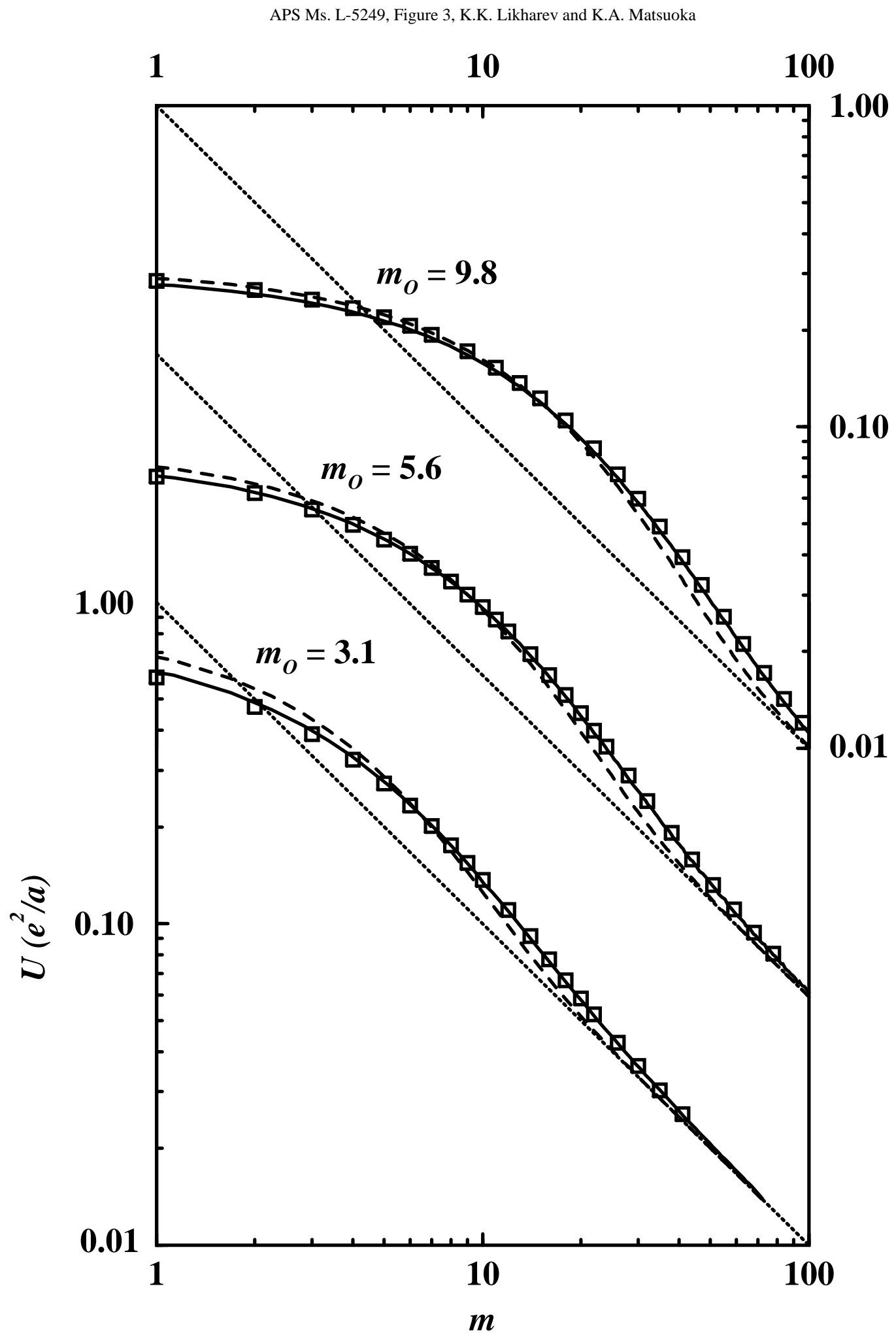




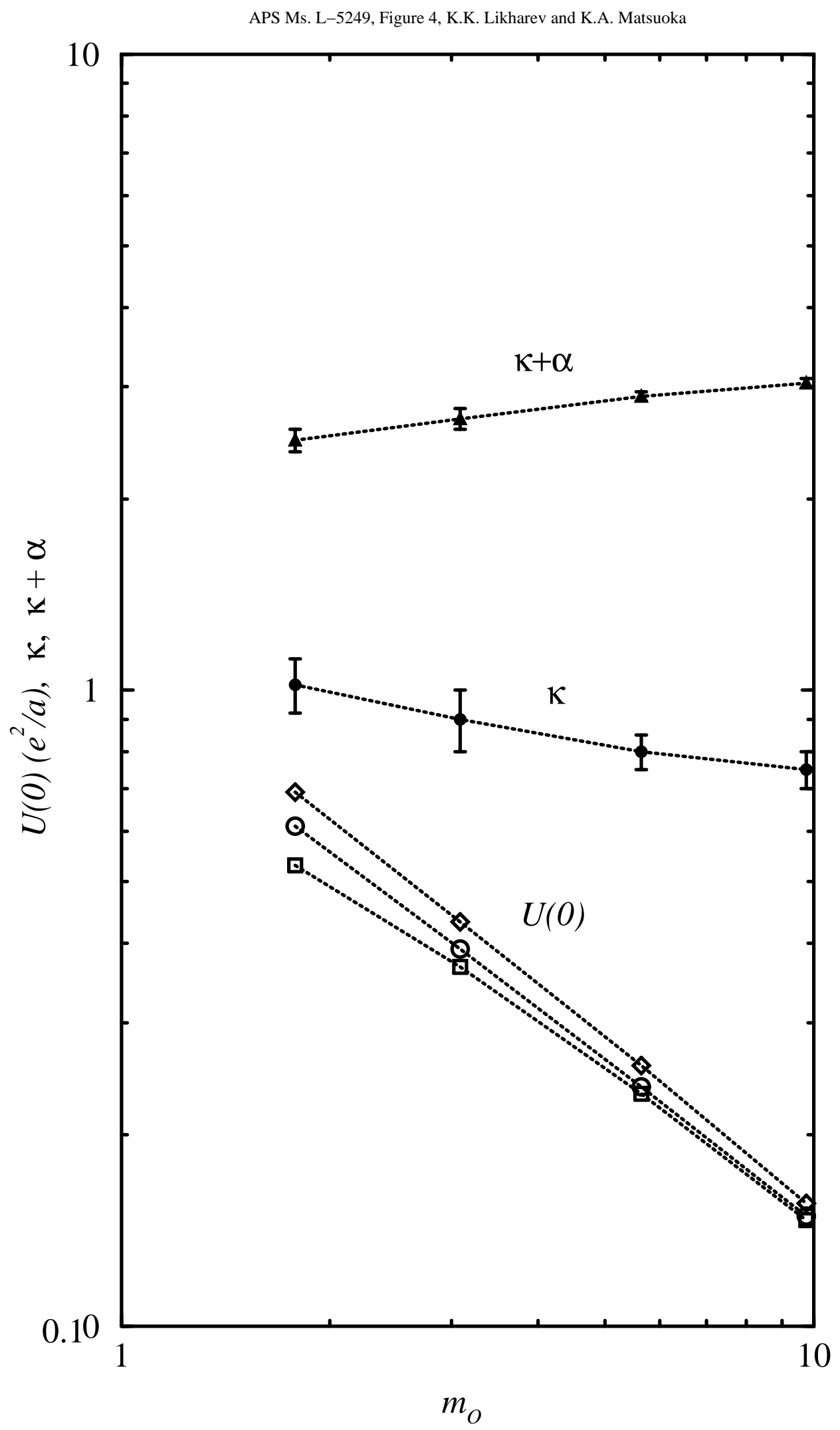

